\title{
Review Study on Effect of Stimulation of Vestibular Apparatus on Postural Muscle Tone in Cerebral Palsy
}

\author{
Mittal $^{1}$, R. \& Narkeesh ${ }^{2}$, A. \\ ${ }^{1}$ Post graduate student, Department of Physiotherapy, Punjabi University, Patiala. \\ ${ }^{2}$ Associate Professor, Department of Physiotherapy, Punjabi University, Patiala.
}

\begin{abstract}
Vestibular Apparatus is a part of inner ear or labyrinth which is responsible for maintaining posture and equilibrium of the body. Vestibular nuclei control selectively the excitatory signals to the antigravity muscles to maintain equilibrium by functioning in association with the pontine reticular nuclei via lateral and medial vestibulospinal tracts. Cerebral palsy is an umbrella-like term used to describe a group of chronic disorders impairing control of movement. A child with cerebral palsy may have difficulty with fine motor tasks, such as writing or cutting with scissors, experience trouble with maintaining balance and walking, or may develop involuntary movements. Aim of the study is to find the effects of induced vestibular stimulation on postural muscle tone in cerebral palsy. Searches of the review study register articles from google.com, pubmed.com, British medical journal.com, Medline, Pedro and online standardized journals. The study was conducted to find the effects of stimulation of vestibular apparatus on postural muscle tone in cerebral palsy. It was concluded that vestibular apparatus plays an important role in maintaining postural tone and vestibular lesions results in disequilibrium with loss of postural control. This review study explains the effect of vestibular apparatus stimulation on postural muscle tone. Vestibular system plays an important role in the achievement of normal motor development and coordination.
\end{abstract}

Key words: Vestibular apparatus, Postural tone, Vestibular nuclei, Postural reflexes, Vestibular apparatus stimulation, Cerebral Palsy

\section{Introduction}

Vestibular apparatus is a part of inner ear or labyrinth which is responsible for maintaining posture and equilibrium of the body. Maintenance of an upright posture involves postural reflexes (which include stretch reflex) which are aided by afferent sensory information from vestibular apparatus and efferent response is to the skeletal muscles. Vestibular apparatus is composed of bony and membranous labyrinth. Membranous labyrinth is a functional part of nervous system which consists of semicircular canals and otolith organs, utricle and saccule that are primarily responsible for equilibrium mechanism (Guyton \& Hall 2006). On standing upright, activity increases in the antigravity postural muscles to counteract the force of gravity this is referred to as Postural Tone (Shumway et al, 2007).

Vestibular inputs activated by a change in head orientation alter the distribution of postural tone in the neck and limbs and have been referred to as Vestibullocollic and vestibular spinal reflexes. Antigravity muscles are the muscles in the body that are tonically active during quiet stance and include 
Soleus and Gastrocnemius, Tibialis Anterior, Gluteus Medius, Tensor Fascia Lata, Iliopsoas, Thoracic Erector Spinae in the trunk along with intermittent activation of abdominals (Basmajian, 1985). The muscle tone in these antigravity muscles is controlled by pontine reticular nuclei that excites the antigravity muscles through the pontine reticulospinal tract and medullary reticular nuclei transmits inhibitory signals to the antigravity anterior motor neuron by way of medullary reticulospinal tract. Vestibular nuclei control selectively the excitatory signals to the antigravity muscles to maintain equilibrium by functioning in association with the pontine reticular nuclei via lateral and medial vestibulospinal tracts. (Guyton \& Hall 2006).

Cerebral palsy is an umbrella-like term used to describe a group of chronic disorders impairing control of movement. The term cerebral refers to the brain's two halves, or hemispheres, and palsy describes any disorder that impairs control of body movement (Madrona, 2001). An individual with cerebral palsy may have difficulty with fine motor tasks, such as writing or cutting with scissors; experience trouble with maintaining balance and walking; or be affected by involuntary movements, such as uncontrollable writhing motion of the hands or drooling. Some children with cerebral palsy are also affected by other medical disorders, including seizures or mental impairment. These children may have difficulty grasping objects, crawling, and walking. They may not get better as they grow up. This condition, that was called Little's disease for many years, is now known as spastic diplegia. It is one of the several disorders that affect control of movement and are grouped together under the term cerebral palsy. Cerebral palsy (ICP) is one of the most common diseases of the nervous system in children. The incidence ranges from 2.5 to 5.9 cases per 1,000 newborns (Madrona, 2001). Cerebral palsy is classified into four broad categories -- spastic, athetoid, ataxic, and mixed forms -- in accordance with the type of movement disturbance.

Spastic cerebral palsy is the most common and affects 70 to 80 percent of patients. The muscles are stiff and permanently contracted. When both legs are affected by spasticity, they may turn in and cross at the knees. This causes a characteristic walking rhythm, known as the scissors gait. Individuals with spastic hemiparesis may also experience hemiparetic tremors, in which uncontrollable shaking affects the limbs on one side of the body. If these tremors are severe, they can seriously impair movement. Athetoid, or dyskinetic, cerebral palsy is characterized by uncontrolled, slow, writhing movements which affect the hands, feet, arms, or legs and, in some cases, the muscles of the face and tongue, causing grimacing or drooling. The movements often increase during periods of emotional stress and disappear during sleep. Patients may also have problems coordinating the muscle movements needed for speech, a condition known as dysarthria. Athetoid cerebral palsy affects about 10 to 20 percent of patients. Ataxic cerebral palsy is a rare form, affecting the sense of balance and depth perception. Affected persons often have poor coordination; walk unsteadily with a wide-based gait, placing their feet unusually far apart and experience difficulty when attempting quick or precise movements, such as writing or buttoning a shirt. These patients may develop intention tremors. 
The ataxic form affects an estimated 5 to 10 percent of cerebral palsy patients. Mixed forms: It is common for patients to have symptoms of more than one of the previous three forms. The most common mixed form may include spasticity and athetoid movements.

The study was aimed to review the studies on the effects of stimulation of vestibular apparatus on postural muscle tone in cerebral palsy.

\section{Methods}

Searches of the review study register articles from google.com, pubmed.com, British medical journal.com, Medline, Pedro and online standardized journals and are presented below.

\section{REVIEWS OF VARIOUS ARTICLES:}

\section{Studies supporting the role of vestibular} apparatus stimulation on postural muscle tone:

Morningstar et al (2005) studied reflex control of spine and posture: a review of literature from chiropractic perspective. In this review there is an attempt to identify the important role the nervous system plays in maintaining reflex control of spine and posture. It concluded that visual and vestibular input as well as joint and soft tissue mechanoreceptors play an important role in the regulation of static upright posture. In this study manual search was done for available relevant textbooks, and a computer search of the medline, mantis, and Index to Chiropractic Literature. Studies were selected if they specifically tested any or all of the postural reflexes either in Earth's gravity or in microgravitational environments. Studies testing the function of each postural component, as well as those discussing postural reflex interactions, were also included in this review. This review of the postural reflex structures and mechanisms adds to the growing body of posture rehabilitation literature. Chiropractic interest in these reflexes may enhance the ability of chiropractic physicians to treat and correct global spine and posture disorders.

Ardic et al (2000) in their study used Galvanic (electrical) vestibular stimulation (GVS) to study the role of the vestibular system in postural control by inducing postural sway in standing subjects. They found that both the paraspinal and gastrocnemius muscles became activated in response to the stimulus. The results of this study suggest that the paraspinal muscles may play a significant role in the frontal plane response to vestibular stimulation during stance in humans. In this study Bineuralbipolar GVS was applied to the skin overlying the mastoid processes of 10 subjects while they stood on a force plate with their eyes closed. The stimulus consisted of a $0.6 \mathrm{~mA}, 5$-pulse sequence. Each pulse lasted for $2 \mathrm{~s}$, followed by $4 \mathrm{~s}$ of rest. The centre of pressure (COP) vs. time for each trial was calculated from the reaction forces and moments. Surface electromyographic (EMG) signals from the paraspinal and gastrocnemius muscles were recorded bilaterally.

Crowne \& Horak (1988) investigated the relationship of vestibular function to motor proficiency, including balance and concluded that impairments of motor proficiency in children with hearing impairments depend on vestibular function. Interventions for motor deficits 
in children with hearing impairments, therefore, must consider vestibular function as well as motor performance. In their study thirteen children with normal hearing and 29 children with hearing impairments, ranging in age from 7 to 13 years, were classified into categories based on vestibular function using neurootologic measures (i.e., vestibulo-ocular reflex function and posturography). Children in each category were tested for motor proficiency using clinical assessment measures (eg, balance, muscle tone, and coordination). The test results indicated that the children with hearing impairments and normal peripheral vestibular function $(n=7)$ exhibited normal motor proficiency, including balance. The children with hearing impairments and loss of peripheral vestibular sensitivity $(\mathrm{n}=19)$ also demonstrated normal motor proficiency, except for balance ability.

Watson (1997) showed EMG responses in the soleus muscle evoked by unipolar galvanic stimulation. They concluded that vestibular activation by clicks can evoke reflex responses in lower-limb muscles and these responses have similar characteristics to the responses evoked by galvanic vestibular stimulation. Reschke et al (1986) showed Vesibulospinal response modification as determined with the H-reflex during the space lab-1 flight. In this study the $\mathrm{H}$ reflex was recorded from the soleus muscle as a method of monosynaptic reflex testing in conjunction with vertical linear acceleration to assess modification of utrculo-saccular function induced through prolonged exposure to microgravity. It showed that early post flight did show an increase in EMG activity and the early inflight $\mathrm{H}$ - reflex amplitude was similar to that recorded at preflight.

Table 1: Summary of Studies supporting the role of vestibular apparatus stimulation on postural muscle tone

\begin{tabular}{|c|c|}
\hline Author & Conclusion \\
\hline $\begin{array}{l}\text { Morningstar } \\
(2005)\end{array}$ & $\begin{array}{l}\text { Studied reflex control of spine and } \\
\text { posture: a review of literature from } \\
\text { chiropractic perspective. It } \\
\text { concluded that visual and vestibular } \\
\text { input as well as joint and soft tissue } \\
\text { mechanoreceptors play an } \\
\text { important role in regulation of } \\
\text { static upright posture. }\end{array}$ \\
\hline Ardic et al (2000) & $\begin{array}{l}\text { The results of this study suggest } \\
\text { that the paraspinal muscles may } \\
\text { play a significant role in the frontal } \\
\text { plane response to vestibular } \\
\text { stimulation (galvanic (electrical) } \\
\text { vestibular stimulation) during } \\
\text { stance in humans }\end{array}$ \\
\hline $\begin{array}{l}\text { Crowne \& Horak } \\
\text { (1988) }\end{array}$ & $\begin{array}{l}\text { Investigated the relationship of } \\
\text { vestibular function to motor } \\
\text { proficiency, including balance and } \\
\text { concluded that impairments motor } \\
\text { proficiency in children with hearing } \\
\text { impairments depends on vestibular } \\
\text { function. }\end{array}$ \\
\hline Watson (1997) & $\begin{array}{l}\text { Concluded that } \text { vestibular } \\
\text { activation by clicks can evoke } \\
\text { reflex responses in lower-limb } \\
\text { muscles and these responses have } \\
\text { similar characteristics to the } \\
\text { responses evoked by galvanic } \\
\text { vestibular stimulation }\end{array}$ \\
\hline $\begin{array}{l}\text { Reschke et al } \\
(1986)\end{array}$ & $\begin{array}{l}\text { Showed vesibulospinal response } \\
\text { modification as determined with } \\
\text { the H-reflex during the space lab-1 } \\
\text { flight. }\end{array}$ \\
\hline
\end{tabular}

Studies supporting effect of stimulation of vestibular apparatus on postural muscle tone in cerebral palsy

Unayik \& Kahiyan (2011) in his article "Down Syndrome: Sensory Integration, Vestibular Stimulation and Neurodevelopmental Therapy Approaches for Children" described that the role of vestibular system is important in the achievement of normal motor development and coordination (Shumway \& and Cook, 1992). The vestibular dysfunction is observed in many developmental disorders as motor discoordination and learning disabilities 
(Horak et al, 1988). The vestibular system is one of the first sensory systems that develop prenatally and is functional at birth due to the completion of its structure anatomically (Shumway \& and Cook, 1992). The vestibular system is particularly important in the development of motor skills, the integration of postural reflexes, forming coordinated eye movements, and visual attention skills, and also in developing inquiringbehavior, and regulating the level of liveliness (Ottenbacher et al, 1983). In contrast to children with isolated vestibular pathology, serious problems are observed in the motor sufficiency of children who demonstrate insufficiency in efficiently organizing visual somatosensorial inputs and normal vestibular inputs for postural control. Therapists who treat children with vestibular dysfunction stimulate the vestibular system with equipment such as swings, scooter boards, and hammocks (Shumway \& and Cook, 1992). Ayres stated that, according to the sensory integration theory, the effect of vestibular stimulation in the central nervous system stems from the plasticity of the nervous system, and that the improvement observed in children in the period following the intervention is continuous because of undeveloped brain plasticity (Ayres, 1979). Ayres pointed out that different head positions and movements are necessary for the stimulation of vestibular receptors, but particularly the horizontal position is more important (Ayres, 1979). Types of vestibular stimulations are to normalization of extensor muscle tone by increasing otolith organ input, linear activities are given in accordance with the order of motor development. These are: bouncing- jumping activities (whilst sitting, kneeling, or standing), linear swinging activities (using platform and T-swing, glider, hammock and barrel swinging in kneeling, standing, sitting, creeping and, prone and supine positions) and other linear activities (jumping or falling onto pillows or mattress in sitting, prone and supine positions). To development of equilibrium reactions by increasing semicircular canal responses, the center of gravity is changed to create disorganization for a short time and thus phasic head movements are made to appear. For this, by moving the support surface, the center of gravity is changed as active or passive and by pushingpulling activities; displacement of the center of gravity is created. These are activities which enable active equilibrium on steep surfaces such as stairs, ramps and unfamiliar surfaces by using equipments such as balance boards, therapy balls and barrel. To lessen the fear of movement or positional change by increasing the weak passing of otolith input, linear vestibular stimulation is applied in tolerable speeds and durations and in unthreatening positions (Fisher, 1989).

Table 2: Summary of Studies supporting effect of stimulation of vestibular apparatus on postural muscle tone in cerebral palsy

\begin{tabular}{ll}
\hline Author & Conclusion \\
\hline Unayik \& Kahiyan & Reported that vestibular \\
(2011) & system is important in the \\
& achievement of normal \\
& motor development and \\
& treatment approach to \\
& cerebral palsy child should \\
& consist of physiotherapy \\
& programs toward the \\
& development of postural \\
& reactions, proprioceptive \\
& and vestibular stimulation \\
& for the development of \\
& visual-motor coordination \\
& and normalization of muscle \\
& tone. \\
\hline Brown (2007) & Reported that in children \\
\hline
\end{tabular}


with cerebral palsy there is abnormal muscle tone, limited movement abilities and problems with tactile and proprioceptive perception due to dysfunction in vestibular sense

Madrona (2001) This study indicated that using the"adeli" method in the rehabilitation of children diagnosed with cerebral palsy stimulates the restarting of the developmental process of the vestibular system and helps in normalization of muscle tone.

Robert et al (1990)

Stated that vestibular system plays an important role in balance and equilibrium and it reinforces the tone of extensor muscles of limbs and trunk thus is responsible for normal gait.

\section{Discussion}

Vestibular apparatus has an important role in postural muscle tone. Morningstar (2005) studied reflex control of spine and posture: a review of literature from chiropractic perspective. In this review there is an attempt to identify the important role the nervous system plays in maintaining reflex control of spine and posture. It concluded that visual and vestibular inputs as well as joint and soft tissue mechanoreceptors play an important role in the regulation of static upright posture. Study of various articles showed that stimulation of vestibular apparatus cause activation of postural tone. Disorders of vestibular apparatus cause difficulty in maintaining balance. The present review study shows that there is further need to find relation between vestibular apparatus and postural muscle tone and further to describe the role of vestibular stimulation in postural tone and its quantification through excitation of monosynaptic reflex in normal adolescents.
The studies of various articles emphasize the role of vestibular system in maintaining normal motor development and co-ordination. In case of cerebral palsy there is abnormal muscle tone, limited movement abilities and problems with tactile and proprioceptive perception. The effectiveness of facilitation of sensory integeration through the vestibular stimulation is a major component of sensory integerative treatment. The vestibular system is particularly important in the development of motor skills, the integration of postural reflexes, forming coordinated eye movements, and visual attention skills, and also in developing inquiringbehavior, and regulating the level of liveliness (Ottenbacher, 1983).

In contrast to children with isolated vestibular pathology, serious problems are observed in the motor sufficiency of children who demonstrate insufficiency in efficiently organizing visual somatosensorial inputs and normal vestibular inputs for postural control. Therapists who treat children with vestibular dysfunction stimulate the vestibular system with equipment such as swings, scooter boards, and hammocks (Shumway \& and Cook, 1992). Ayres stated that, according to the sensory integration theory, the effect of vestibular stimulation in the central nervous system stems from the plasticity of the nervous system, and that the improvement observed in children in the period following the intervention is continuous because of undeveloped brain plasticity (Ayres, 1972). Further research is required to use vestibular stimulation in habituating the neuromuscular loop resulting in a quantifiable difference in muscle tone. With this new techniques 
that assist with motor control deficiency found in cerebral palsy can be invented.

\section{Conclusion}

The review study concluded that there is supporting literature about the relation between functioning of vestibular apparatus and postural muscle tone. This review shows that stimulation of vestibular apparatus causes increase in activation of postural muscle tone. Vestibular system plays an important role in the achievement of normal motor development and coordination. In contrast to children with isolated vestibular pathology, serious problems are observed in the motor sufficiency of children who demonstrate insufficiency in efficiently organizing visual somatosensorial inputs and normal vestibular inputs for postural control. Stimulation of vestibular receptors is used to normalization of extensor muscle tone by increasing otolith organ input; linear activities are given in accordance with the order of motor development.

There are a number of precautions to consider the vestibular stimulation such as as a result of over stimulation, sensory overload occurs and this results in organization dysfunctions in the central nervous system. Therefore, over stimulating should be avoided, and before, during, and after vestibular stimulation, the child should be checked for evidence of over stimulation or under stimulation and allowed to determine his own speed. The over inhibition of the brainstem is the greatest potential harm resulting in seizures, cyanosis, and depression in vital functions. In children with hypertonicity, a counter effect in the form of more tone increase may occur. Sensory stimulation response is different in each child, and the child should be checked carefully at this time (Fisher, 1989). This study concluded that family education within early intervention programs for infants should give importance to the prone position and the variety of movement, and should consist of physiotherapy programs toward the development of postural reactions, proprioceptive and vestibular stimulation, the perception of the sense of touch and body awareness, ocular control and the development of visual-motor coordination.

Rehabilitation of Children Diagnosed with Cerebral Palsy Using "Adeli" Suit is based on Dynamic Proprioceptive Correction (Madrona, 2001). Development of the brain and the spinal cord is significantly influenced by the interceptive impulses from the ligamentomuscular apparatus and vestibular system. Structures of the vestibular system are one of the major zones processing impulses from proprioceptors. Factors injuring/damaging the central nervous system (CNS) significantly compromise functions at all levels of the vestibular system. This results in (motor) dysfunction in all CNS levels responsible for the formation and control of movement. This study indicated that using the"Adeli" method in the rehabilitation of children diagnosed with Cerebral Palsy stimulates the restarting of the developmental process of the vestibular system and helps in normalization of muscle tone. Gradually, particularly after the 2nd and 3rd rehabilitation session, the function within the semicircular canals and otolithic organs normalized and manifested in decreased spasticity. Improvement in vestibular system functioning resulted in diminished 
hyperkinesis. Application of "Adeli" Suit therapy influences the flow of the afferent prioprioceptive impulses which in turn stimulate the development of the vestibular system.

The vestibular system integrates all other functional systems, which stimulate the postnatal development of the CNS. The CNS determines the normalization of motor development, speech and cognitive development. Sensory Integrative Treatment was developed out of the extensive research of A. Jean Ayres, an occupational therapist with a strong interest in the sensory systems and sensory integrative dysfunction (Ayres 1972). Therapy provides controlled sensory input (especially input from the vestibular, tactile, and proprioceptive systems) in a way that allows the child to make an adaptive response that integrates the sensations and enhances the organization of the brain.

\section{References}

Ardic, F.N., Latt, L.D., Redfern, M.S. 2000. Paraspinal muscle response to electrical vestibular stimulation. Acta. Otolaryngol. 120: $39-46$.

Ayres, A.J. 1979. Sensory integration and the child. Los Angeles: Western Psychological Services". taken from Unayik M. and Kahiyan H. 2011. Down syndrome: sensory integeration, vestibular stimulation and Neurodevelopmental therapy approaches for children. Intern. Encyclop. of Rehab.. In: J.H. Stone, M. Blouin, editors Link: http://cirrie.buffalo.edu/encyclopedia/en/ article/en/article/48/

Basmajen, 1985. taken from Anne Shumway, Cook, Marjorie H. Woollacott (2007), "Motor control: Translating research into clinical practice". $3^{\text {rd }}$ edition, Publisher: Philadelphia: Lippincott Williams ann Wilkins. pp 160-165
Link:http://filescadbs.org/2000000353_9f 7fca079e/2_vestibular_dbrownpdf

Christropher J.D, Billy, L., Luu, K., van den, D., Inglis, J.T, Blouin, J.S 2009. FrequencySpecific modulation of vestibular evoked sway Responses in humans" $J$. Neurophysiol., 103(2): 1048-1056

Crowne T.K, Horak F.B 1988. Motor proficiency associated with vestibular deficits in children with hearing impairments" Acta Otolaryngol 68(10): 1493-149.

Fisher, 1989. Taken from Unayik M. and Kahiyan H.(2011), " Down syndrome: sensory integeration, vestibular stimulation and Neurodevelopmental therapy approaches for children" International Encyclopedia of Rehabilitation In: J.H. Stone, MBlouin, editors

Link:http://cirrie.buffalo.edu/encyclopedi a/en/article/en/article/48/

Guyton, A.C, Hall, J.E. 2006. Textbook of Medical Physiology. $11^{\text {th }}$ edition, Elsevier founder publication. 692-697

Horak, F.B., Shumway-Cook, A, Crowe, TK, Black, F.O. 1988. Vestibular function and motor proficiency of children with impaired hearing, or with learning disability and motor impairments". Developmental Medicine and Child Neurology: 64-79. taken from Unayik M. and Kahiyan H. 2011. Down syndrome: sensory integeration, vestibular stimulation and Neurodevelopmental therapy approaches for children" International Encyclopedia of Rehabilitation. In: J.H. Stone, M. Blouin, editors Link: http://cirrie.buffalo.edu/encyclopedia/en/ article/en/article/48/

Levinson, H.N. 1990. The Diagnostic Value Of Cerebellar-Vestibular Tests In detecting Learning disabilities, dyslexia, attention deficit disorders, Perceptual and Motor skills" J. Neuropsych. 71: 67-82.

Lin, S.R, Gage, R.J 1990. Neurological control system for normal Gait" 2(1): 1-13.

Madrona 2001. http://www.healingarts.org/children/cp/cpoverview.htm

Brown, D. 2007. The vestibular sense" 
Morningstar, M.W., Pettibon, B.R, Schlappi. H., Schlappi, M. and Ireland, T.V. 2005. Reflex control of spine and posture: A review of literature from a Chiropractic perspective and osteopathy. J. Phys. Occup. Ther., 16(2): 1-21.

Ottenbacher, K.J., Petersen, P. 1983. The Efficacy of Vestibular Stimulation as a Form of Specific sensory Enrichment. Quantitative Review of the Literature. Clinical Pediatrics, 428-433 taken from Unayik, M. and Kahiyan, H. 2011. Down syndrome: sensory integeration, vestibular stimulation and Neurodevelopmental therapy approaches for children" International Encyclopedia of Rehabilitation In: J.H. Stone, M. Blouin, editors. Link: http://cirrie.buffalo.edu/encyclopedia/en/ article/en/article/48/

Reschke, M.F., Anderson, D.J., Homick, J.L 1986. Vestibulospinal Response Modification as determined with the H- Reflex during the space lab-1 flight. J. Appl. Physiol., 90(3): 997-1006.

Shumway, Cook, A., Woollacott, M.H. 2007. Motor control: Translating research into clinical practice. $3^{\text {rd }}$ edition, Publisher: Philadelphia: Lippincott Williams and Wilkins, 160-165
Shumway and Cook, A. 1992. Role of the Vestibular System in Motor Development: Theoretical and Clinical Issues. In: Forssberg, H., Hirschfeld, H. (Editors), Movement Disorders in Children. Medicine and Sport Science. Basel, Karger taken from Unayik M. and Kahiyan H. 2011. Down syndrome: sensory integeration, vestibular stimulation and Neurodevelopmental therapy approaches for children" International Encyclopedia of Rehabilitation In: J.H. Stone, M.Blouin, editors

Link:http://cirrie.buffalo.edu/encyclopedi a/en/article/en/article/48/

Unayik, M. and Kahiyan, H. 2011. Down syndrome: sensory integeration, vestibular stimulation and Neurodevelopmental therapy approaches for children" International Encyclopedia of Rehabilitation In: Stone, J.H. \& Blouin, $\quad \mathrm{M}$, editors Link:http://cirrie.buffalo.edu/encyclopedi a/en/article/en/article/48/

Watson, S.R.D. 1997. EMG responses in the soleus muscle evoked by unipolar galvanic Stimulation." J. Electromyog. Mot. Cont. 15(6): 476-483. 